\title{
MicroRNA-497 acts as a tumor suppressor in gastric cancer and is downregulated by DNA methylation
}

\author{
JICHAO LIU, YONGSHUANG LI, YING ZOU, JIAKUI ZHANG, \\ JIAXIANG AN, JIAO GUO, MINGHUI MA and DONGQIU DAI \\ Department of Gastrointestinal Surgery, The Fourth Affiliated Hospital, \\ China Medical University, Shenyang, Liaoning 110032, P.R. China
}

Received December 14, 2016; Accepted May 18, 2017

DOI: $10.3892 /$ or.2017.5698

\begin{abstract}
Gastric cancer (GC) is one of the most common malignant tumors in the world and microRNAs (miRNAs) play an important role in GC. In this study, we found miR-497 played an important role and served as a novel biomarker in GC. Quantitative real-time PCR (qRT-PCR) was used to measure the miR-497 expression in GC cell lines and 86 paired GC samples and we also analyzed its correlation with GC clinicopathological parameters. A series of cellular function experiments were applied to validate the effects of miR-497 on GC. In addition, methylation-specific PCR (MSP) was applied to detect the gene methylation status. Finally, the correlation between miR-497 and the target gene was analyzed by western blotting assay. miR-497 was reduced obviously in GC cells and tissues and significantly associated with the pathologic stage. Low expression of miR-497 significantly inhibited the proliferation, invasion and migration of GC cell lines and accelerated apoptosis. Moreover, we found that the aberrant expression of miR-497 may be ascribed to DNA methylation. microRNA.org and luciferase reporter assay suggested that RAF1 was a direct target of miR-497 in GC. This study suggested that miR-497 could serve as a tumor suppressor and a potential early diagnostic marker of GC by targeting Raf-1 proto-oncogene, serine/threonine kinase (RAF1).
\end{abstract}

\section{Introduction}

Gastric cancer (GC) is a highly malignant digestive tract tumor, and most GC patients have a poor prognosis due to lack of an early diagnostic indicator (1). Thus, it is crucial to find early effective biological indicators of GC to improve the survival rate of GC patients. For the past few years, a large

Correspondence to: Dr Dongqiu Dai, Department of Gastrointestinal Surgery, The Fourth Affiliated Hospital, China Medical University, 4 Chongshan Road, Shenyang, Liaoning 110032, P.R. China

E-mail: daidq2016@163.com

Key words: miR-497, gastric cancer, RAF1, DNA methylation, tumor suppressor amount of reports confirmed that miRNAs are closely related to GC genesis and development (2-8).

miRNAs are short endogenous RNAs and they have no function of protein coding. Numerous studies have shown that dysregulated expression of miRNA participates in GC malignant phenotype. Wu et al illuminated that high expression of miR-15a in GC led to Bmi-1 protein inactivation and had a negative effect on overall survival of GC patients (9). Seok et al proved that hypoxia could activate the function of miR-382, whereafter, inhibited the expression of PTEN gene and played a role of angiogenic oncogene in GC (10). Previous studies also suggested that miRNAs had a critical function of human GC progression $(11,12)$. However, the mechanism of aberrant regulation of miRNA in GC remained largely unknown. Latest studies found that the abnormal expression of miRNA might be ascribed to promoter hypermethylation. For instance, Yin et al considered that the inhibition of miR-33b was mediated by DNA methylation in GC and the lowerexpression of miR-33b contribute to advanced GC clinical stage (13). Li et al indicated that promoter hypermethylation was responsible for the downregulation of miR-335 and overexpression of miR-335 could inhibit GC cells invasion and metastasis by targeting RASA1 (14). This evidence suggested that miRNAs might serve as a novel biomarker and played an important role in gastric cancer.

miR-497 was first reported in breast cancer and is located on chromosome 17p13.1 (15). Numerous studies had reported that miR-497 was closely related to various carcinomas. For example, Yan et al demonstrated that miR-497 negatively regulated VEGFA and inhibited hepatocellular carcinoma metastasis (16). Wang et al proved that miR-497 repressed angiogenesis of ovarian cancer by retrograde regulated PI3K/AKT pathway (17). However, Jiang et al found that miR-497 acted as an oncogene in colorectal cancer (18). Wald et al also proved that the expression of miR-497 was increased in human head and neck carcinoma (19). In fact, there is some controversy about the effect of miR-497 in different tumors and the unique expression of miR-497 in different cancers might be tissue-specific. Despite the many reports that miR-497 has an important role in various human carcinomas, the regulatory mechanism of miR-497 in GC was still unclear.

In this investigation, we validated that miR-497 was reduced significantly in GC and repressed GC cell proliferation, 

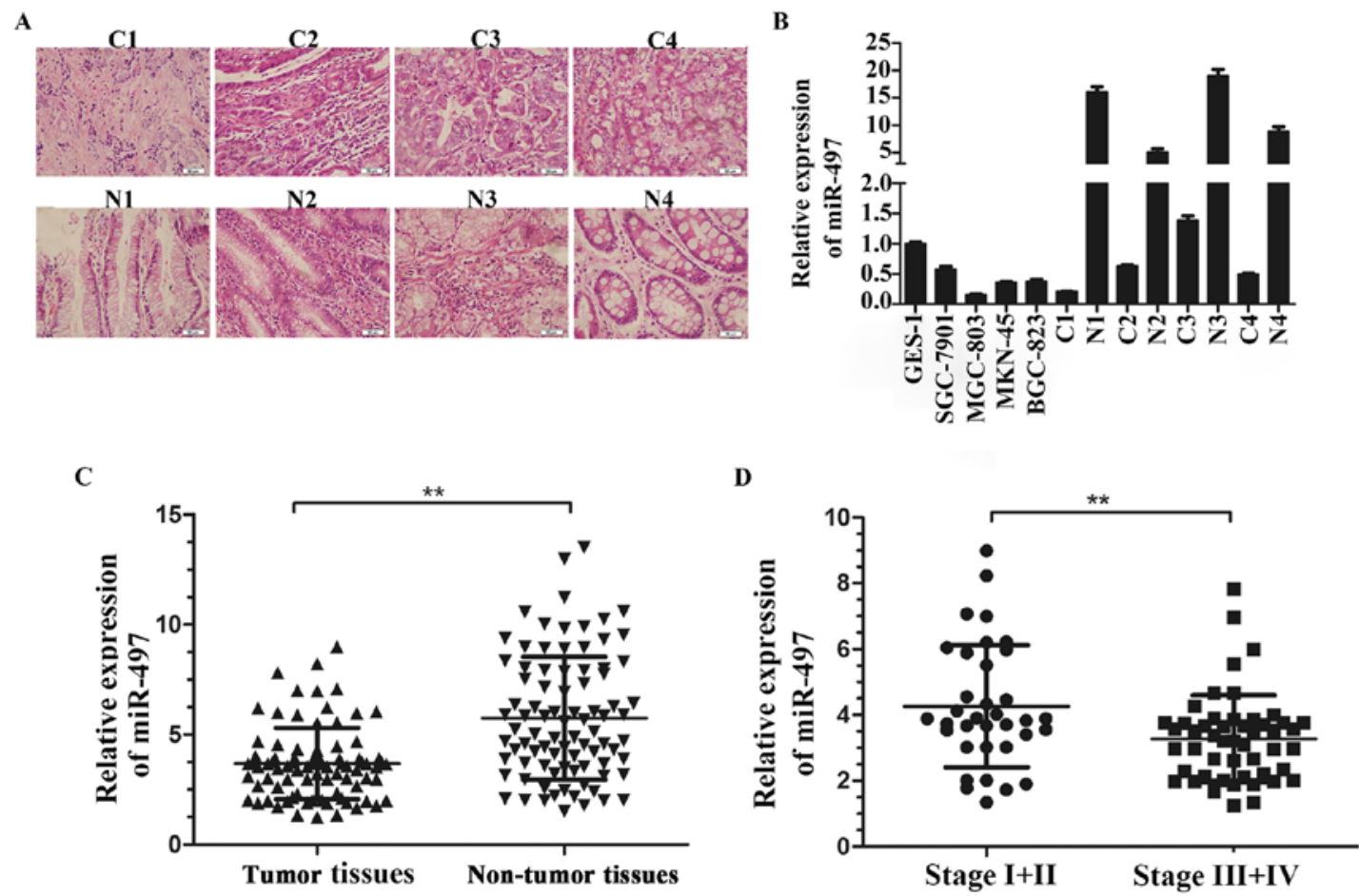

Figure 1. miR-497 is downregulated in GC cells and tissues. (A) Four paired GC samples stained with hematoxylin and eosin (H\&E). C, cancer tissues; $\mathrm{N}$, normal tissues. (B) The relative expression of miR-497 in GC cell lines, and a normal gastric cell line and four paired GC samples. (C) Expression of miR-497 in 86 paired GC samples was measured by qRT-PCR. (D) The expression level of miR-497 is related to advanced clinical stage in GC $\left({ }^{* *} \mathrm{P}<0.01\right)$.

invasion and metastasis. Further research indicated that gene promoter hypermethylation might contribute to dysregulation of miR-497. We also revealed that miR-497 mediated GC progression by retrograde targeting RAF1.

\section{Materials and methods}

Gastric cancer cell lines and clinical samples. Human GC cell lines SGC-7901, MGC-803, MKN-45, BGC-823 and a normal gastric mucomembrane cell line GES-1 were obtained from Chinese Academy of Sciences, Shanghai Institutes for Cell Resource Center. Cells were cultivated in RPMI-1640 medium (Corning, Manassas, VA, USA) containing 10\% FBS (Corning). All cells were maintained in $5 \% \mathrm{CO}_{2}$ at $37^{\circ} \mathrm{C}$. Eighty-six cases of GC tissues were collected from 2013 to 2015 at Cancer Research Institute of China Medical University, tissues were histologically confirmed and stored at $-80^{\circ} \mathrm{C}$ after surgical removal until further analysis. The study was approved by the Ethics Committee of China Medical University.

Cell treatments. For cell transfection, cells were grown in 6 -well culture plates for $24 \mathrm{~h}$, then, $50 \mu \mathrm{M}$ miR-497 mimics or mimics control (mimics-NC) (GenePharma, Shanghai, China) was added into the culture medium using Lipofectamine 3000 reagent (Invitrogen, Calsbad, CA, USA) following the manufacturer's instructions. For 5-Aza-dC (Sigma, St. Louis, MO, USA) and trichostatin A (TSA) (Sigma) treatment assay, SGC-7901 and MGC-803 cells were first incubated in 6-well culture dishes for $24 \mathrm{~h}$. Then, for 5-Aza-dC treatment group, 0 , $0.5,1$ or $1.5 \mu \mathrm{mol} / 15-\mathrm{Aza}-\mathrm{dC}$ were mixed with the cell culture medium for $72 \mathrm{~h}$. For TSA treatment group, cells were treated with $0.3 \mu \mathrm{mol} / 1 \mathrm{TSA}$ for $24 \mathrm{~h}$. For combination of drugs intervention group, $1 \mu \mathrm{mol} / 15$-Aza-dC was first mixed with the medium for $48 \mathrm{~h}$ and then $0.3 \mu \mathrm{mol} / 15$-Aza-dC was added for another $24 \mathrm{~h}$.

RNA extraction and $q R T-P C R$. RNA Extraction kit (Takara, Dalian, China) was used to purify total RNA from GC cells and tissues. Reverse transcription and qRT-PCR were performed using SYBR Green qRT-PCR kit (GenePharma). U6 SnRNA was identified as an internal reference. The U6 primers used were 5'-ATTGGAACGATACAGAGAAGATT-3' and 5'-GGAACGCTTCACGAATTTG-3', the miR-497 primers used were 5'-GACGACCTCAGCAGCACACT-3' and 5'-CAG AGCAGGGTCCGAGGTA-3'. We conducted the PCR assay according to our previous report (20) and the experiment was repeated in triplicate.

DNA extraction and MSP. A GenElute ${ }^{\mathrm{TM}}$ Mammalian Genomic DNA Miniprep kit (Sigma) was used to separate the genetic DNA from the paired GC tissues. Then, purified DNA was conducted with a bisulfite treatment using the EZ DNA Methylation-Gold kit ${ }^{\mathrm{TM}}$ (Zymo Research, Irvine, CA, USA), this assay was carried out according to the manufacturer's instructions. MethPrimer 1.0 was used to design MSP primer for methylation-specific PCR (MSP). The methylated primers used were 5'-TTTGTTTTTGTGTTAGAGAGGGTTC-3' (forward) and 5'-TTTGTTTTTGTGTTAGAGAGGGTTC-3' (reverse), the unmethylated primers used were 5'-TGTTTTT GTGTTAGAGAGGGTTTG-3' (forward) and 5'-ACTAAC AATACAACTACATCCCATA-3' (reverse). We conducted the PCR assay according to Jia et al (21), this experiment was repeated in triplicate. 
Cell proliferation assay and cell apoptosis assay. Cell Counting Kit-8 (Dojindo, Kumamato, Japan) was used to detect the proliferation rates of GC cells. First, 3,000 SGC-7901 and MGC-803 cells were incubated into 96-well plates for $24 \mathrm{~h}$. Then, $10 \mu \mathrm{l}$ CCK-8 was added into each well, proliferation rates of GC cells were surveyed at $0,24,48$ and $72 \mathrm{~h}$ after transfection. This experiment was repeated for three times. Annexin V-PE/7AAD Apoptosis Detection kit (KeyGen, Jiangsu, China) was applied to perform the cell apoptosis assay according to the manufacturer's instructions and this experiment was repeated in triplicate.

Cell migration and invasion assays. Transfected cell lines were grown in 6-well plates for $24 \mathrm{~h}$ and linear wounds were scratched on the confluent cells using a $200-\mu 1$ pipette tip. The serum-free medium was replaced by cell culture and we used an inverted microscope to visualize migrated cells and wound healings. For cell invasion assay, Matrigel-coated membrane matrix (Dojindo) was smeared onto the upper chamber for $24 \mathrm{~h}$, then, $1 \times 10^{5}$ transfected cells were grown in the upper chambers with serum-free medium and the lower chambers was added with medium containing $10 \%$ FBS. Twenty-four hours later, non-invasive cells were removed using a cotton tip and the invasive cells were stained using crystal violet (Tiangen, Beijing, China). Images were taken using an inverted microscope. Each sample was repeated in triplicate.

Western blot analysis and histology. Proteins were extracted from GC cells and clinical samples using RIPA buffer (Beyotime, Shanghai, China) and BCA Protein assay kit (Takara) was used to measure the protein concentration. Then, $30 \mu \mathrm{g}$ proteins were added into $10 \%$ SDS-PAGE (Takara) and polyvinyl fluoride membranes (Beyotime) were conducted as a transmembrane. Membranes were blocked for $2 \mathrm{~h}$ with BSA (Sigma) and kept at $4^{\circ} \mathrm{C}$ overnight with anti-RAF1 antibody and we set an internal control using $\beta$-actin. The dilution ratio was $1: 1,000$. Thereafter, the membrances were incubated with secondary antibody (Sigma) at room temperature for $2 \mathrm{~h}$. At last, images were determined using an enhanced chemiluminescence system. For histology, first, buffered formalin was used to fix tissues and then we embedded tissues in paraffin. Second, paraffin-embedded blocks were cut into $3 \mu \mathrm{m}$ slices and stained with hematoxylin and eosin (H\&E).

Luciferase reporter assay. miR-497 mimics or mimics control (mimics-NC), reporter construct or control vector (GenePharma) were transfected in GC cells for $24 \mathrm{~h}$. Dual Luciferase assay kit (KeyGen) was used to detect luciferase activity. The ratio of luciferase intensities was calculated and normalized to controls. Each sample was carried out in triplicate.

Statistical analysis. We used SPSS 19.0 to conduct statistical analyses and Student's t-test, an ANOVA test and $\chi^{2}$ test was utilized to analyze $\mathrm{P}$-values. $\mathrm{P}<0.05$ was considered to indicate a statistically significant difference (two-sided).

\section{Results}

miR-497 is decreased in GC cell lines and tissues. We first examined the expression level of miR-497 in four GC cell
Table I. Clinicopathological characteristics of patients with gastric cancer (GC).

\begin{tabular}{|c|c|c|c|c|}
\hline \multirow[b]{2}{*}{ Parameter } & \multirow[b]{2}{*}{$\begin{array}{c}\text { Total } \\
\text { samples }(\mathrm{n}) \\
\mathrm{n}=86\end{array}$} & \multicolumn{2}{|c|}{ miR-497 expression } & \multirow[b]{2}{*}{ P-value } \\
\hline & & $\begin{array}{c}\text { Low } \\
\text { expression } \\
(\%)\end{array}$ & $\begin{array}{c}\text { High } \\
\text { expression } \\
\text { (5) }\end{array}$ & \\
\hline Age (years) & & 48 & 38 & 0.681 \\
\hline$\geq 60$ & 23 & $12(52.2)$ & $11(47.8)$ & \\
\hline$<60$ & 63 & $26(57.1)$ & $27(42.9)$ & \\
\hline Sex & & & & 0.310 \\
\hline Male & 66 & $39(59.1)$ & $27(40.9)$ & \\
\hline Female & 20 & $9(45.0)$ & $11(55.0)$ & \\
\hline Location & & & & 0.557 \\
\hline Proximal & 12 & $5(41.7)$ & $7(58.3)$ & \\
\hline Body & 20 & $12(60.0)$ & $8(40.0)$ & \\
\hline Distal & 54 & $31(57.4)$ & $23(42.6)$ & \\
\hline Tumor size & & & & 0.730 \\
\hline $\mathrm{T} 1-\mathrm{T} 2$ & 38 & $22(57.9)$ & $16(42.1)$ & \\
\hline T3-T4 & 48 & $26(54.2)$ & $22(45.8)$ & \\
\hline Borrmann type & & & & 0.459 \\
\hline Borrmann $1+2$ & 11 & $5(45.5)$ & $6(54.5)$ & \\
\hline Borrmann $3+4$ & 75 & $43(57.3)$ & $32(42.7)$ & \\
\hline Grade & & & & 0.298 \\
\hline $\begin{array}{l}\text { Well and } \\
\text { moderately } \\
\text { differentiated }\end{array}$ & 31 & $15(48.4)$ & $16(51.6)$ & \\
\hline $\begin{array}{l}\text { Poorly } \\
\text { differentiated }\end{array}$ & 55 & $33(60.0)$ & $22(40.0)$ & \\
\hline TNM stage & & & & 0.014 \\
\hline $\mathrm{I}+\mathrm{II}$ & 35 & $14(66.7)$ & $21(33.3)$ & \\
\hline III+IV & 51 & $34(55.8)$ & $17(44.2)$ & \\
\hline
\end{tabular}

lines (SGC-7901, MGC-803, MKN-45, BGC-823) and four paired GC tissues (Fig. 1A) and GES-1 was used as a standard control, the data suggested miR-497 was significantly downregulated in GC cell lines compared with GES-1, similarly, GC tissues showed low expression of miR-497 compared to the corresponding normal tissues (Fig. 1B). To further assess miR-497 expression in GC, we measured the expression level of miR-497 in 86 pairs of GC tissues and corresponding normal tissues. The data demonstrated that miR-497 was significantly downregulated in GC tissues compared to adjacent normal tissues (Fig. 1C). The data proved that low expression of miR-497 was frequent in gastric cancer and might be relevant to GC genesis.

The low expression of miR-497 in GC indicates advanced clinical stage. To illuminate the relevance between miR-497 expression and GC clinicopathological parameters, we separated the patients into two groups according to the mean level of miR-497. Our data showed low miR-497 expression was apt to have an advanced GC TNM stage $(\mathrm{P}=0.014)$. However, no obvious relationship was found between miR-497 expression and tumor size or location (Table I and Fig. 1D). The result 

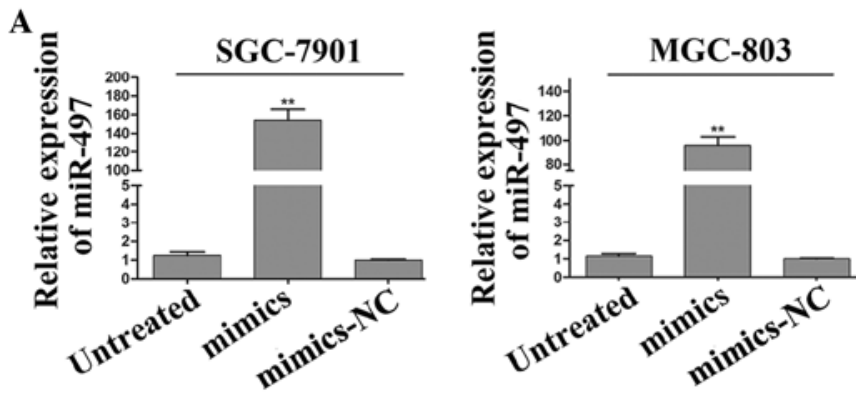

C
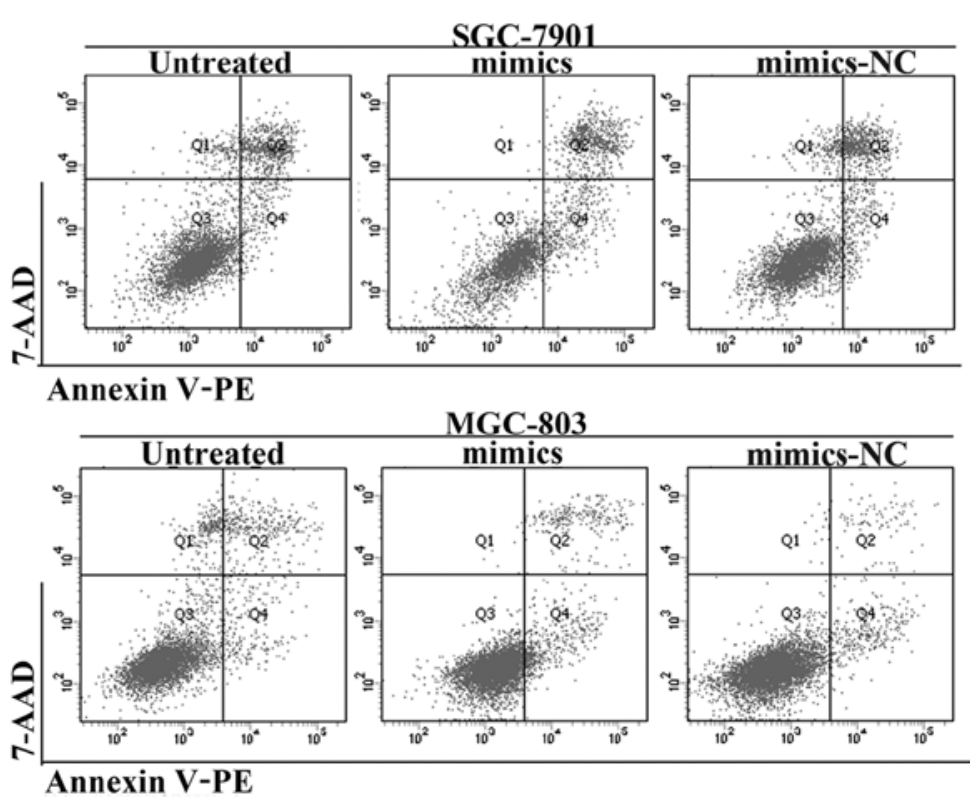
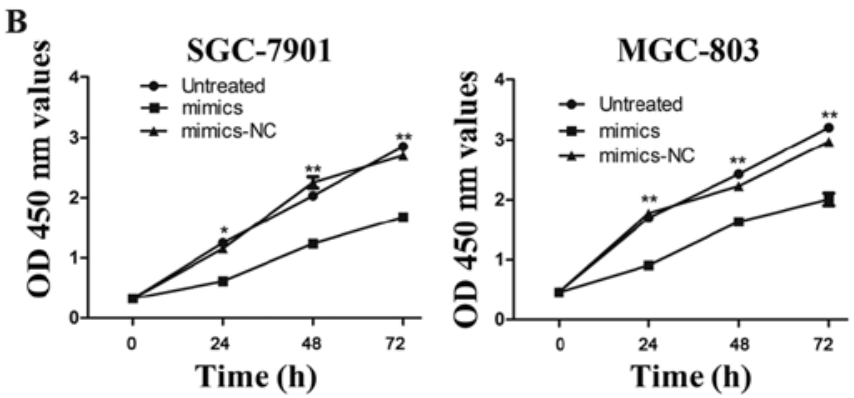

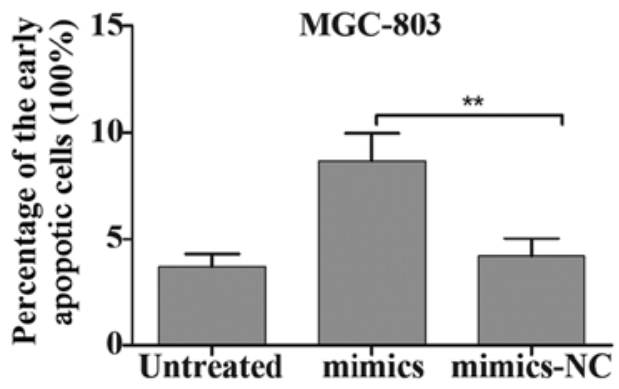

Figure 2. Ectopic expression of miR-497 regulates SGC-7901 and MGC-803 proliferation and apoptosis. (A) The transfection efficiency of miR-497 in SGC-7901 and MGC-803 cells. (B) The proliferation ability of GC cells transfected with miR-497 mimics or mimics-NC was detected by CCK-8 at different time-points. (C) miR-497 significantly accelerated GC cell apoptosis. ${ }^{*} \mathrm{P}<0.05 ;{ }^{* *} \mathrm{P}<0.01$.

indicated that miR-497 might be involved in GC malignant progression.

Effects of miR-497 on GC cell proliferation and apoptosis. In order to analyze the role of miR-497 in GC tumorigenesis and progression, miR-497 mimics were transfected in SGC-7901 and MGC- 803 cells using Lipo 3000 . To measure the transfection efficiency, qRT-PCR was applied to determine miR-497 expression level after cell transfection, the data exhibited that miR-497 was upregulated $154.33 \pm 11.50$ times and $96.74 \pm 6.82$ times in SGC-7901 and MGC-803 cells (Fig. 2A). As shown in CCK- 8 growth assay, restoration of miR-497 obviously reduced the growth of SGC-7901 and MGC-803 cells (Fig. 2B). Our data proved that miR-497 obviously inhibted GC cell proliferation. To further explore the effect of miR-497 on GC cell apoptosis, cell apoptosis assay was carried out to count early apoptosis cells. The miR-497 mimics control group showed few early apoptotic cells $(5.32 \pm 0.93 \%$ in SGC-7901 and $4.24 \pm 0.82 \%$ in MGC-803), whereas mimics-transfected group revealed a higher percentage of early apoptotic cells $(12.66 \pm 2.31 \%$ in SGC-7901 and $8.11 \pm 1.27 \%$ in MGC-803), Quadrant IV of flow cytometry chart is early apoptotic cells (Fig. 2C). In general, we showed that miR-497 might inhibit GC cell survival by promoting cell apoptosis.
miR-497 inhibits GC cell migration and invasion in vitro. To explore whether miR-497 could regulate the metastasis ability of GC, a wound healing assay was performed to measure the GC cell migration ability. As showed in Fig. 3A, a significant difference was detected in a wound healing assay. Overexpression of miR-497 in SGC-7901 and MGC-803 cells induced a great reduction of cell migration. Moreover, transwell invasion assay also demonstrated that upregulation of miR-497 dramatically inhibited cell migration in GC cells (Fig. 3B). The result was consistent with our viewpoint that the reduction of miR-497 in GC might contribute to tumor metastasis in gastric carcinogenesis.

Downregulation of miR-497 is associated with promoter hyper-methylation. In order to investigate the regulatory mechanism of low expression of miR-497 in GC, human genome database was used to find $\mathrm{CpG}$ islands around miR-497 (Fig. 4A) and MSP primers were conducted to analyze the methylation status of the miR-497 gene promoter in GC cell lines (SGC-7901, MGC-803), the result showed that SGC-7901 and MGC-803 cells possessed a hypermethylation status of miR-497 gene promoter compared with GES-1. We also analyzed the methylation status of SGC-7901 and MGC-803 cells treated with 5-Aza-dC, the result showed that 5-Aza-dC 
A
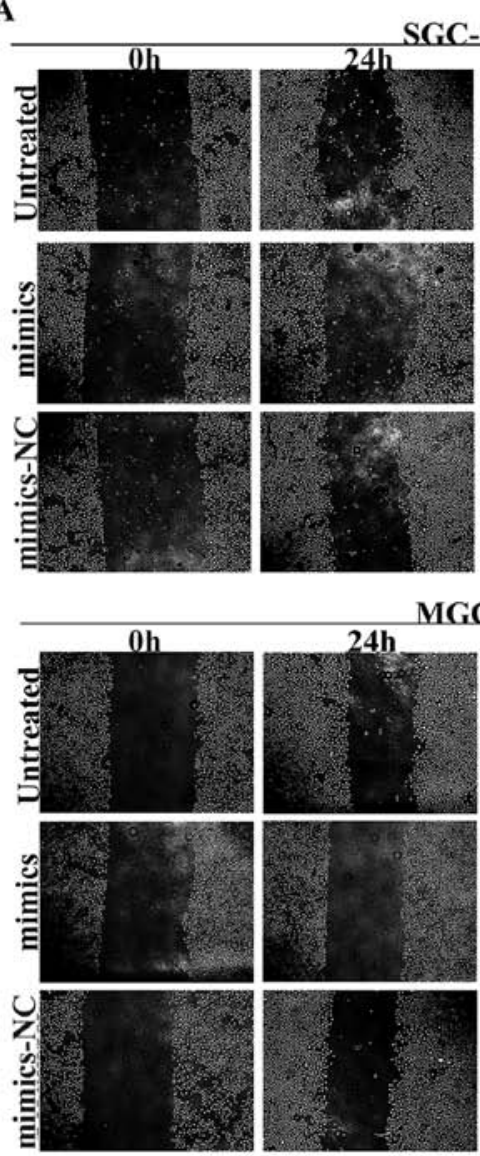

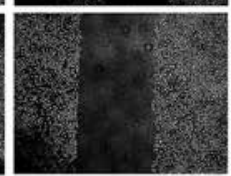

SGC-7901

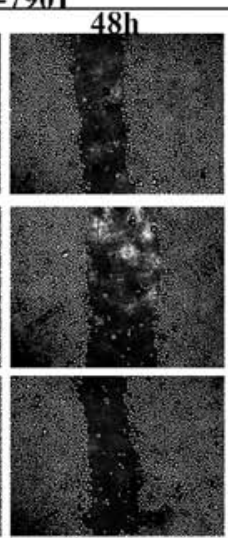

GC-803
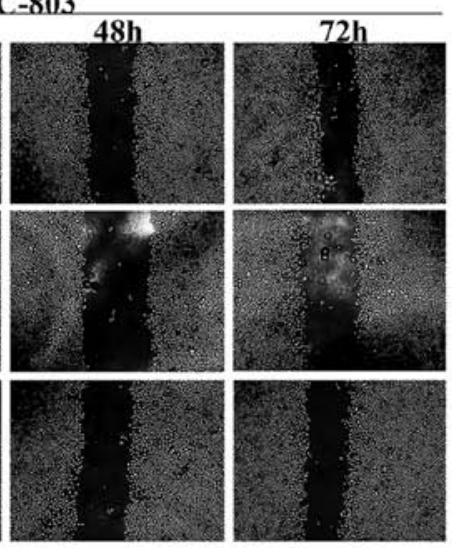

B
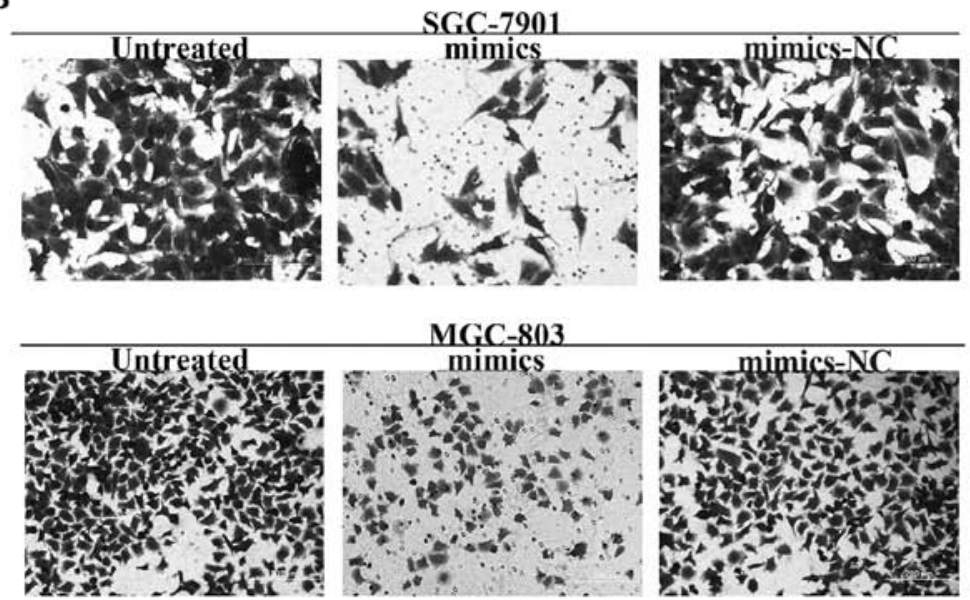
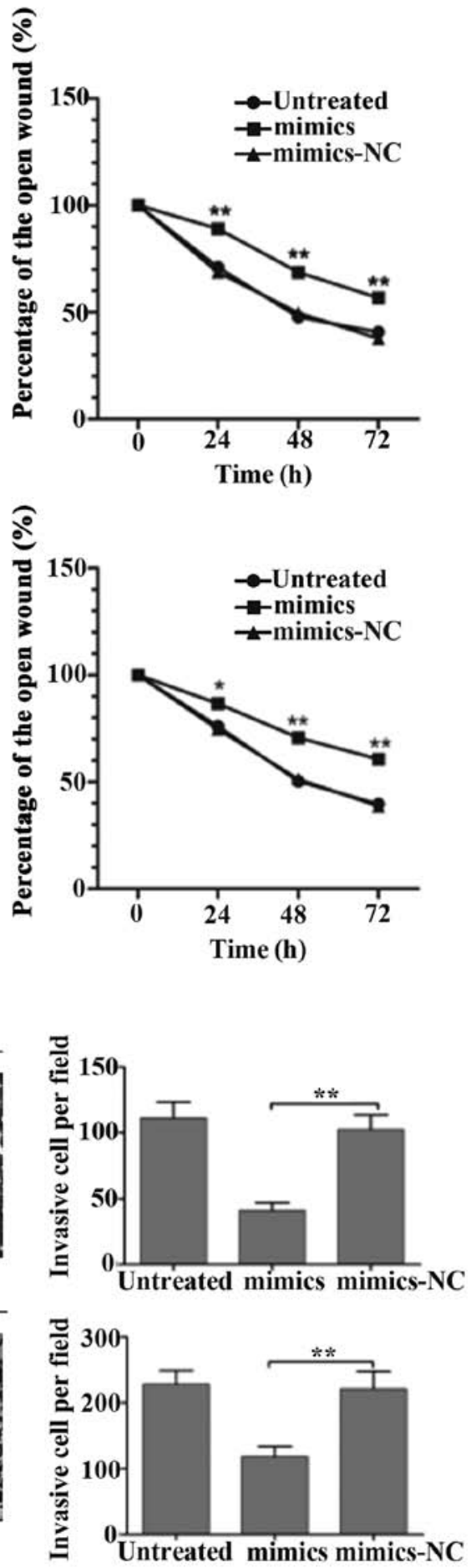

Figure 3. Effect of miR-497 on GC cell migration and invasion. (A) Overexpression of miR-497 enhances the migration ability of GC cells. (B) miR-497 obviously suppressed GC cells invasion. ${ }^{*} \mathrm{P}<0.05 ;{ }^{* *} \mathrm{P}<0.01$.

could change the hypermethylation status of miR-497 gene promoter in SGC-7901 and MGC-803 cells (Fig. 4B). To further confirm whether the reduction of miR-497 in GC was due to DNA methylation, DNA methylation inhibitor 5-Aza-dC and histone deacetylase inhibitor TSA were used to treat GC cells and the expression level of miR-497 was detected immediately. The result proved that miR-497 expression was increased after treatment with 5-Aza-dC and miR-497 expression was restored the most when the 5-Aza-dC concentration was set to $1.5 \mu \mathrm{M}$.
However, there was no obvious change of miR-497 expression after treatment with TSA alone (Fig. 4C). To further illuminate the relationship between low expression of miR-497 and gene promoter methylation in $\mathrm{GC}$, we measured the methylation status of the miR- 497 promoter of 8 cases of paired GC tissues. Eight cases of GC samples were divided into two groups according to the expression level of miR-497. The result showed that the low miR-497 expression group possessed a higher hyper-methylation percentage $(75 \%, 3 / 4)$ opposed to 
A

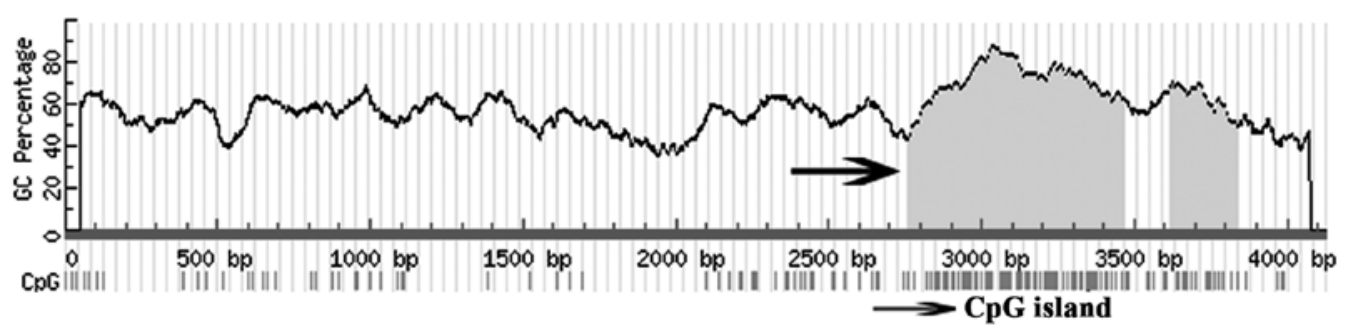

B

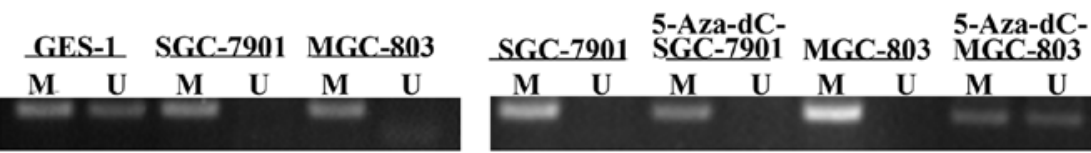

C
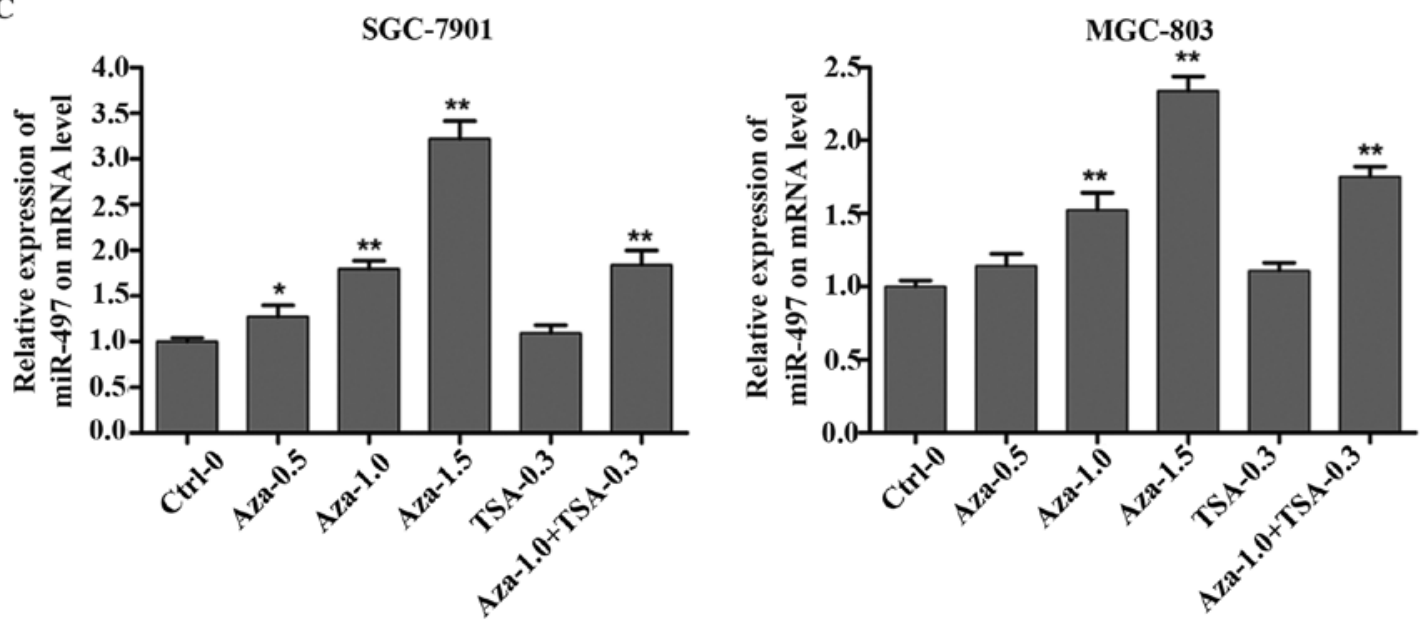

D

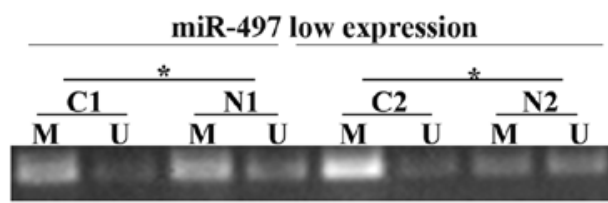

miR-497 high expression

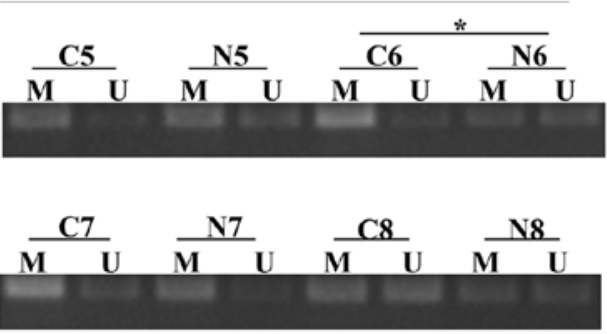

Figure 4. Low expression of miR-497 in GC is related to DNA methylation. (A) Arrow is CpG island location. (B) Methylation status in GC cell lines and a normal gastric cell line, methylation status in GC cell lines treated with 5-Aza-CdR or not. (C) 5-Aza-CdR obviously enhanced the expression level of miR-497. Ctrl, black control group; Aza, 5-Aza-CdR treatment group; TSA, TSA treatment group. Aza+TSA, combination of drugs intervention group. (D) Methylation status in 8 paired GC tissues. M, methylated; U, unmethylated. C, cancer tissues; $\mathrm{N}$, normal tissues. The hypermethylation samples are marked. ${ }^{*} \mathrm{P}<0.05 ;{ }^{* *} \mathrm{P}<0.01$.

the high miR-497 expression group (25\%, 1/4) (Fig. 4D). The result indicated that the low miR-497 expression in GC might be due to gene promoter hyper-methylation.

miR-497 targets RAF1 in GC. miRNAs play their biologic role through downregulating their target genes. microRNA. org was utilized for finding target genes of miR-497. The result showed that miR-497 has a binding site on the RAF1 mRNA 3'-UTR (Fig. 5A). Futhermore, luciferase reporter assay showed that miR-497 mimics could induce an obviously reduction of the luciferase activity of wild-type RAF1-3'-UTR. However, miR-497 mimics could not affect the luciferase activity of mutant RAF1-3'-UTR (Fig. 5B). The result further indicated that miR-497 has a binding site on the RAF1 mRNA 3'-UTR. To confirm that miR-497 performs biological functions in GC by targeting RAF1, western blotting was applied to measure the protein level of RAF1 in SGC-7901 and MGC-803 cells. We concluded that the protein level of RAF1 was obviously downregulated in miR-497 mimics-treated cells compared to scramble-treated and untreated cells (Fig. 5C). We further measured the expression of RAF1 protein in low miR-497 expression GC patients and we found that RAF1 was obviously increased in GC tissues compared to the adjacent normal tissues (Fig. 5D), which was consistent with the results in GC cells. 

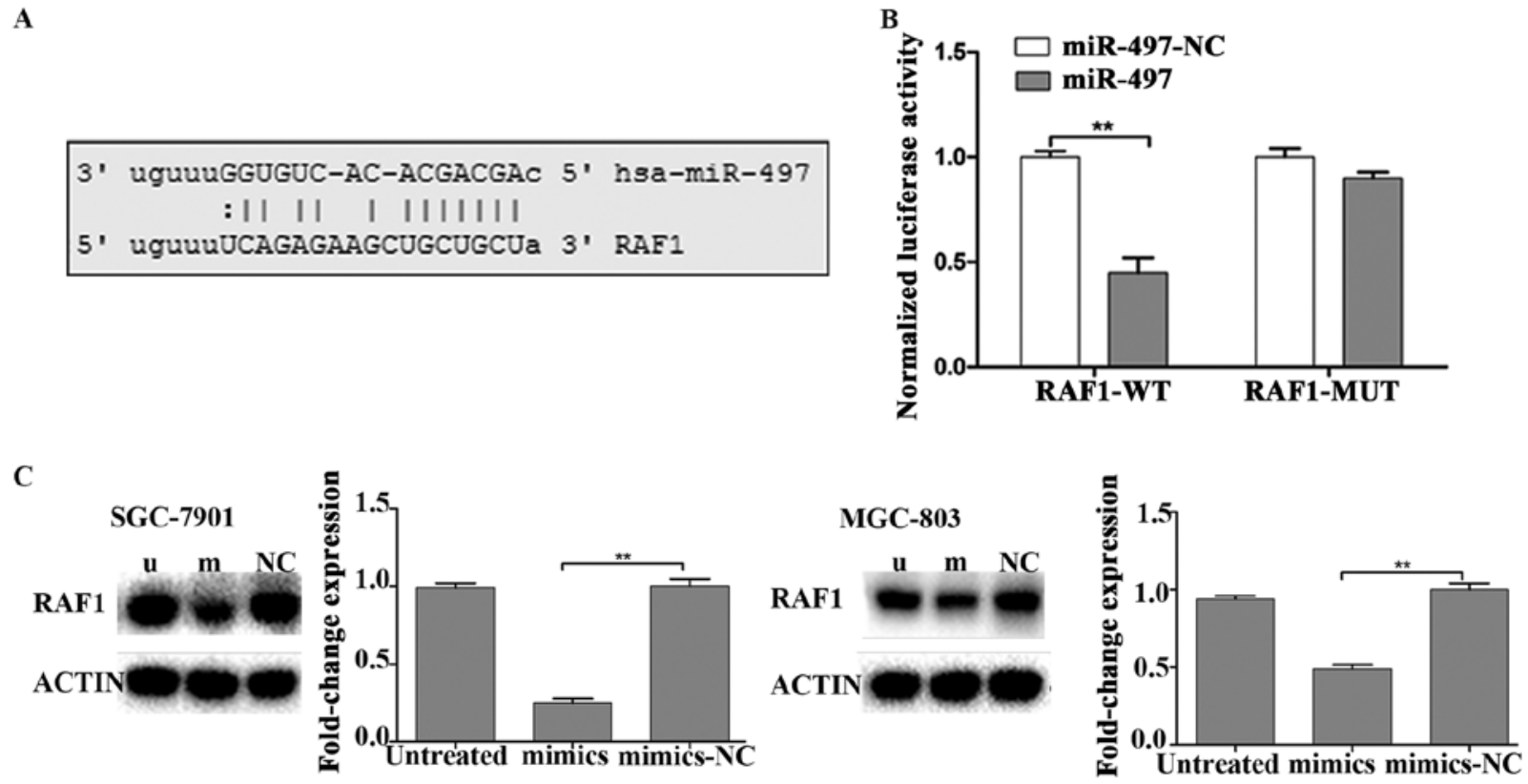

D
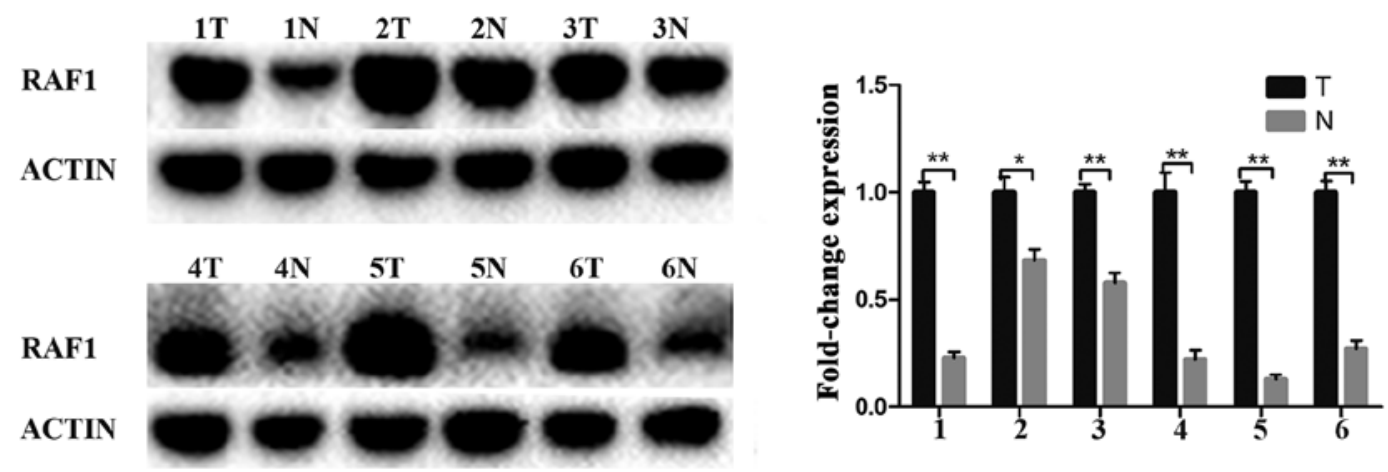

Figure 5. miR-497 directly targets RAF1. (A) The predicted miR-497 binding site on the RNF1 mRNA 3'-UTR. (B) Luciferase activity of wild-type (WT-UTR) or mutant (MUT-UTR). (C) Western blot analysis of RAF1 expression in miR-497-transfected SGC-7901 and MGC-803 GC cells. u, untreated; m, mimics; NC, mimics control. (D) RAF1 protein expression level in 6 paired GC tissues. T, cancer tissues; $\mathrm{N}$, normal tissues. ${ }^{*} \mathrm{P}<0.05 ;{ }^{* *} \mathrm{P}<0.01$.

\section{Discussion}

Previous studies suggested that miRNAs functioned as oncogenes or tumor suppressors in various carcinomas and miRNAs played important roles in GC carcinogenesis and progression (22-24). However, the possible molecular mechanism of miRNAs in regulating GC occurrence and progression was unknown. Here we proved that miR-497 was obviously downregulated in GC cells and tissues and the most important was that reduction of miR-497 was significantly associated with advanced clinical stage. Moreover, we demonstrated that restoration of miR-497 induced GC cell apoptosis and inhibited GC cell proliferation, migration, and invasion. To further clarify the regulatory mechanism of miR-497 in GC, we found that downregulation of miR-497 might due to DNA methylation and RAF1 was regarded as a direct target of miR-497 in GC.

Yan et al first indicated that miR-497 was downregulated in human breast cancer (15), which was further confirmed by Lehmann $e t$ al and $\mathrm{Li}$ et al $(25,26)$. Han et al showed that low expression of miR-497 in lung cancer could inhibit lung cancer cell growth through negatively regulating cyclin E1 expression (27). Xu et al found that miR-497 was decreased in ovarian cancer and might act as a potential therapeutic target. However, Wald et al reported that miR-497 was upregulated in human head and neck carcinoma (19). Jiang et al also found that miR-497 was overexpressed in colorectal cancer (18). The aberrant expression of miR-497 in different tumors might be ascribed to tissue specificity. A recent study proved that miR-497 was downregulated in multidrug-resistant SGC-7901 cell lines and a latest research indicated that miR-497 was downregulated in GC tissues $(28,29)$. However, the molecular mechanism and the possible regulatory pathway of downregulation of miR-497 in GC remain unclear. For this reason, we detected the expression of miR-497 in GC cell lines and tissues. The data indicated that miR-497 was significantly decreased in GC cell lines as well as in GC tissues compared to the normal control. Furthermore, we explored the clinicopathological correlations of miR-497 in GC tissues and the data proved that patients with low miR-497 expression possessed an advanced clinical stage. All the data suggested miR-497 might participate in GC tumorigenesis and progression. 
Accumulating studies illuminated that miRNAs were closely related to tumorigenesis, however, the exact mechanism involved were unknown. To demonstrated the effect of miR-497 on GC carcinogenesis and progression, we conducted cellular function tests including cell proliferation assay, cell invasion assay and cell apoptosis assay. The result proved that restoration of miR-497 obviously inhibited GC cell proliferation, migration, and invasion, the result also indicated that overexpression of miR-497 significantly induced GC cell apoptosis. Apoptosis can be seen as a stage-dependent process from its induction to early, intermediate and late-stage apoptotic events. Early apoptosis is represented by changes to, and ultimate loss of, the mitochondrial membrane potential. Our study indicated that low expression of miR-497 in GC was a frequent event and might play crucial roles in GC tumorigenesis. Recently, numerous studies proved that downregulation of miRNAs was associated with epigenetic modifications, including DNA methylation and histone modification (30,31). Among them, DNA methylation was one of the most frequent epigenetic changes in human cancers and might be responsible for downregulation of miRNAs in various carcinomas $(14,32)$. In this study, we detected that miR-497 was located in a CpG island on chromosome 17P13.1 and the MSP analysis showed that GC cell lines possessed a hyper-methylation status compared with the normal control. Moreover, GC cells treated with 5-Aza-dC showed DNA promoter demethylation, whereas cells treated with TSA demonstrated no obvious alterations of DNA methylation status. Thus, we concluded that downregulation of miR-497 might be inactivated by hyper-methylation in GC.

Moreover, our study found RAF1 harboring miR-497 binding site and RAF1 was considered to be a potential target of miR-497. Particularly, RAF1 gene was located in 3p25.2 chromosome and its aberrant expression was reported to be associated with tumor malignant biological properties (33-35). Activation of the RAS-RAF pathway was responsible for poor prognosis of colorectal cancer patients (36). Moreover, hyperactivation of RAF1 could induce cancer cell resistance to multidrugs through RAF1-MEK1-ERK/AKT pathway and might have predictive value in treatments of cancers (37). These studies suggested that RAF1 could act as a critical parameter for tumor genesis and progression. Furthermore, Wang et al showed that miR-195 inhibited cell proliferation through blocking the expression of RAF1 in thyroid cancer (38). Liu et al indicated that miR-7-5p suppressed cell proliferation through directly targeting RAF1 in glioblastoma (39). Accumulating evidence has proved that RAF1 is upregulated in various carcinomas, however, the relationship between miRNAs and RAF1 in GC was still unclear. In this report, we found the protein level of RAF1 in miR-497 mimicstransfected GC cells was significantly lower than normal control. The result proved that miR-497 might serve as a tumor suppressor gene in GC by targeting RAF1.

In conclusion, our results indicated that miR-497 had a tumoral suppression function through targeting RAF1 in GC and decreased expression of miR-497 might due to DNA hypermethylation. GC patients who possessed low miR-497 level were apt to have an advanced GC clinical stage. Restoration of miR-497 obviously inhibited GC cell proliferation, migration and invasion. The result proved that miR-497 could act as a novel biomarker and therapeutic target in gastric cancer.

\section{Acknowledgements}

This study was supported in part by a grant from the National Natural Science Foundation of China (no. 30572162), the Liaoning Province Science and Technology Plan Project (no. 2013225021) and the Natural Science Foundation of Liaoning Province (no. 201602817).

\section{References}

1. Li G, Wang Z, Ye J, Zhang X, Wu H, Peng J, Song W, Chen C, Cai S, He Y, et al: Uncontrolled inflammation induced by AEG-1 promotes gastric cancer and poor prognosis. Cancer Res 74: 5541-5552, 2014.

2. Jiang C, Chen X, Alattar M, Wei J and Liu H: MicroRNAs in tumorigenesis, metastasis, diagnosis and prognosis of gastric cancer. Cancer Gene Ther 22: 291-301, 2015.

3. Yang L, Liang H, Wang Y, Gao S, Yin K, Liu Z, Zheng X, Lv Y, Wang L, Zhang CY, et al: MiRNA-203 suppresses tumor cell proliferation, migration and invasion by targeting Slug in gastric cancer. Protein Cell 7: 383-387, 2016.

4. Zhu M, Wang M, Yang F, Tian Y, Cai J, Yang H, Fu H, Mao F, Zhu W, Qian $\mathrm{H}$, et al: miR-155-5p inhibition promotes the transition of bone marrow mesenchymal stem cells to gastric cancer tissue derived MSC-like cells via NF- $\kappa \mathrm{B}$ p65 activation. Oncotarget 7: 16567-16580, 2016.

5. Zhang X, Ni Z, Duan Z, Xin Z, Wang H, Tan J, Wang G and Li F: Overexpression of E2F mRNAs associated with gastric cancer progression identified by the transcription factor and miRNA co-regulatory network analysis. PLoS One 10: e0116979, 2015.

6. Zhang X, Tang J, Zhi X, Xie K, Wang W, Li Z, Zhu Y, Yang L, $\mathrm{Xu} \mathrm{H}$ and $\mathrm{Xu} \mathrm{Z}$ : miR-874 functions as a tumor suppressor by inhibiting angiogenesis through STAT3/VEGF-A pathway in gastric cancer. Oncotarget 6: 1605-1617, 2015.

7. Kim JG, Kim TO, Bae JH, Shim JW, Kang MJ, Yang K, Ting AH and Yi JM: Epigenetically regulated MIR941 and MIR1247 target gastric cancer cell growth and migration. Epigenetics 9: 1018-1030, 2014

8. Matsuo M, Nakada C, Tsukamoto Y, Noguchi T, Uchida T, Hijiya N, Matsuura K and Moriyama M: miR-29c is downregulated in gastric carcinomas and regulates cell proliferation by targeting RCC2. Mol Cancer 12: 15, 2013.

9. Wu C, Zheng X, Li X, Fesler A, Hu W, Chen L, Xu B, Wang Q, Tong A, Burke S, et al: Reduction of gastric cancer proliferation and invasion by miR-15a mediated suppression of Bmi-1 translation. Oncotarget 7: 14522-14536, 2016.

10. Seok JK, Lee SH, Kim MJ and Lee YM: MicroRNA-382 induced by HIF- $1 \alpha$ is an angiogenic miR targeting the tumor suppressor phosphatase and tensin homolog. Nucleic Acids Res 42: 80628072, 2014

11. Xu X, Wang W, Su N, Zhu X, Yao J, Gao W, Hu Z and Sun Y: miR-374a promotes cell proliferation, migration and invasion by targeting SRCIN1 in gastric cancer. FEBS Lett 589: 407-413, 2015.

12. Sakamoto N, Naito Y, Oue N, Sentani K, Uraoka N, Zarni Oo H, Yanagihara K, Aoyagi K, Sasaki H and Yasui W: MicroRNA-148a is downregulated in gastric cancer, targets MMP7, and indicates tumor invasiveness and poor prognosis. Cancer Sci 105: 236-243, 2014.

13. Yin H, Song P, Su R, Yang G, Dong L, Luo M, Wang B, Gong B, Liu C, Song W, et al: DNA methylation mediated down-regulating of MicroRNA-33b and its role in gastric cancer. Sci Rep 6: $18824,2016$.

14. Li Z, Li D, Zhang G, Xiong J, Jie Z, Cheng H, Cao Y, Jiang M, Lin L, Le Z, et al: Methylation-associated silencing of MicroRNA-335 contributes tumor cell invasion and migration by interacting with RASA1 in gastric cancer. Am J Cancer Res 4: 648-662, 2014.

15. Yan LX, Huang XF, Shao Q, Huang MY, Deng L, Wu QL, Zeng YX and Shao JY: MicroRNA miR-21 overexpression in human breast cancer is associated with advanced clinical stage, lymph node metastasis and patient poor prognosis. RNA 14: 2348-2360, 2008.

16. Yan JJ, Zhang YN, Liao JZ, Ke KP, Chang Y, Li PY, Wang M, Lin JS and He XX: miR-497 suppresses angiogenesis and metastasis of hepatocellular carcinoma by inhibiting VEGFA and AEG-1. Oncotarget 6: 29527-29542, 2015. 
17. Wang W, Ren F, Wu Q, Jiang D, Li H and Shi H: MicroRNA-497 suppresses angiogenesis by targeting vascular endothelial growth factor A through the PI3K/AKT and MAPK/ERK pathways in ovarian cancer. Oncol Rep 32: 2127-2133, 2014.

18. Jiang Y, Meng Q, Qi J, Shen H and Sun S: miR-497 promotes metastasis of colorectal cancer cells through Nrdp1 inhibition. Tumour Biol 36: 7641-7647, 2015.

19. Wald AI, Hoskins EE, Wells SI, Ferris RL and Khan SA: Alteration of microRNA profiles in squamous cell carcinoma of the head and neck cell lines by human papillomavirus. Head Neck 33: 504-512, 2011.

20. Liu J, Zhang J, Li Y, Wang L, Sui B and Dai D: miR-455-5p acts as a novel tumor suppressor in gastric cancer by down-regulating RAB18. Gene 592: 308-315, 2016.

21. Jia H, Zhang Z, Zou D, Wang B, Yan Y, Luo M, Dong L, Yin H, Gong B, Li Z, et al: MicroRNA-10a is down-regulated by DNA methylation and functions as a tumor suppressor in gastric cancer cells. PLoS One 9: e88057, 2014.

22. Lei H, Zou D, Li Z, Luo M, Dong L, Wang B, Yin H, Ma Y, Liu C, Wang F, et al: MicroRNA-219-2-3p functions as a tumor suppressor in gastric cancer and is regulated by DNA methylation. PLoS One 8: e60369, 2013.

23. Tsai MM, Wang CS, Tsai CY, Chen CY, Chi HC, Tseng YH, Chung PJ, Lin YH, Chung IH, Chen CY, et al: MicroRNA-196a/-196b promote cell metastasis via negative regulation of radixin in human gastric cancer. Cancer Lett 351: 222-231, 2014

24. Zhou X, Xia Y, Li L and Zhang G: miR-101 inhibits cell growth and tumorigenesis of Helicobacter pylori related gastric cancer by repression of SOCS2. Cancer Biol Ther 16: 160-169, 2015.

25. Lehmann U, Streichert T, Otto B, Albat C, Hasemeier B, Christgen H, Schipper E, Hille U, Kreipe HH and Länger F: Identification of differentially expressed microRNAs in human male breast cancer. BMC Cancer 10: 109, 2010.

26. Li D, Zhao Y, Liu C, Chen X, Qi Y, Jiang Y, Zou C, Zhang X, Liu S, Wang X, et al: Analysis of miR-195 and miR-497 expression, regulation and role in breast cancer. Clin Cancer Res 17: 1722-1730, 2011

27. Han Z, Zhang Y, Yang Q, Liu B, Wu J, Zhang Y, Yang C and Jiang Y: miR-497 and miR-34a retard lung cancer growth by co-inhibiting cyclin E1 (CCNE1). Oncotarget 6: 13149-13163, 2015.

28. Zhu W, Zhu D, Lu S, Wang T, Wang J, Jiang B, Shu Y and Liu P: miR-497 modulates multidrug resistance of human cancer cell lines by targeting BCL2. Med Oncol 29: 384-391, 2012.

29. Li W, Jin X, Deng X, Zhang G, Zhang B and Ma L: The putative tumor suppressor microRNA-497 modulates gastric cancer cell proliferation and invasion by repressing eIF4E. Biochem Biophys Res Commun 449: 235-240, 2014.
30. Ye Z, Shen N, Weng Y, Li K, Hu L, Liao H, An J, Liu L, Lao S and Cai S: Low miR-145 silenced by DNA methylation promotes NSCLC cell proliferation, migration and invasion by targeting mucin 1. Cancer Biol Ther 16: 1071-1079, 2015.

31. Hirata H,Hinoda Y, Shahryari V, Deng G, Tanaka Y, Tabatabai ZL and Dahiya R: Genistein downregulates onco-miR-1260b and upregulates SFRP1 and Smad4 via demethylation and histone modification in prostate cancer cells. Br J Cancer 110: 1645-1654, 2014.

32. Cao J, Song Y, Bi N, Shen J, Liu W, Fan J, Sun G, Tong T, He J, Shi Y, et al: DNA methylation-mediated repression of miR-886-3p predicts poor outcome of human small cell lung cancer. Cancer Res 73: 3326-3335, 2013.

33. Cianci P, Tono V, Sala A, Locatelli L, Carta C, Rizzari C, Biondi A and Selicorni A: A boy with Burkitt lymphoma associated with Noonan syndrome due to a mutation in RAF1. Am J Med Genet A 161A: 1401-1404, 2013.

34. Ren G, Liu X, Mao X, Zhang Y, Stankiewicz E, Hylands L, Song R, Berney DM, Clark J, Cooper C, et al: Identification of frequent BRAF copy number gain and alterations of RAF genes in Chinese prostate cancer. Genes Chromosomes Cancer 51: 1014-1023, 2012.

35. Albers C, Illert AL, Miething C, Leischner H, Thiede $M$, Peschel C and Duyster J: An RNAi-based system for lossof-function analysis identifies Raf1 as a crucial mediator of BCR-ABL-driven leukemogenesis. Blood 118: 2200-2210, 2011.

36. Li X, Stevens PD, Liu J, Yang H, Wang W, Wang C, Zeng Z, Schmidt MD, Yang M, Lee EY, et al: PHLPP is a negative regulator of RAF1, which reduces colorectal cancer cell motility and prevents tumor progression in mice. Gastroenterology 146: 1301-12.e1, 10, 2014.

37. Xu ZH, Hang JB, Hu JA and Gao BL: RAF1-MEK1-ERK/AKT axis may confer NSCLC cell lines resistance to erlotinib. Int J Clin Exp Pathol 6: 1493-1504, 2013

38. Wang F, Jiang C, Sun Q, Yan F, Wang L, Fu Z, Liu T and Hu F: miR-195 is a key regulator of Raf 1 in thyroid cancer. Onco Targets Ther 8: 3021-3028, 2015.

39. Liu Z, Liu Y, Li L, Xu Z, Bi B, Wang Y and Li JY: miR-7-5p is frequently downregulated in glioblastoma microvasculature and inhibits vascular endothelial cell proliferation by targeting RAF1. Tumour Biol 35: 10177-10184, 2014. 